\title{
A solitary pulmonary nodule due to eosinophilic granuloma
}

\author{
G.P.M. ten Velde*, F.B.J.M. Thunnissen**, \\ J.M.A. van Engelshoven+, E.F.M. Wouters*
}

A solitary pulmonary nodule due to eosinophilic granuloma. G.P.M. ten Velde, F.B.J.M. Thunnissen, J.M.A. van Engelshoven, E.F.M. Wouters. CERS Journals Ltd 1994.

ABSTRACT: A symptomless 42 year old man with a smoking history of 40 packyears presented with a solitary pulmonary nodule highly suggestive of lung cancer.

Wedge resection proved it to be an eosinophilic granuloma. No other localizations of eosinophilic granuloma were found, and none developed during a $3 \mathrm{yr}$ follow-up.

Eur Respir J., 1994, 7, 1539-1540.
Department of *Pulmonology, **Pathology and +Radiology, University Hospital Maastricht, Maastricht, The Netherlands.

Correspondence: G.P.M. ten Velde, Dept of Pulmonology, University Hospital Maastricht, P.O. Box 5800, 6202 AZ Maastricht, The Netherlands

Keywords: Eosinophilic granuloma solitary pulmonary nodule

Received: October 291993

Accepted after revision February 141994
The finding of a solitary pulmonary nodule (SPN) on a chest radiograph in a symptomless patient remains a difficult problem for diagnosis and treatment. In patients over 40 yrs of age, $40-50 \%$ of these lesions are malignant $[1,2]$. Decision analysis based on Bayes theorem and four variables (age, history of cigarette smoking, diameter of the nodule, and prevalence of malignancy in SPNs) [3] can help in making the decision between the two therapeutic options: thoracotomy, or wait and see.

We report a case strongly suggestive of a malignant SPN, which proved to be an eosinophilic granuloma of the lung. This has been reported only once previously [4].

\section{Case report}

A 42 year old man underwent an examination for life insurance. His past history was remarkable for a 40

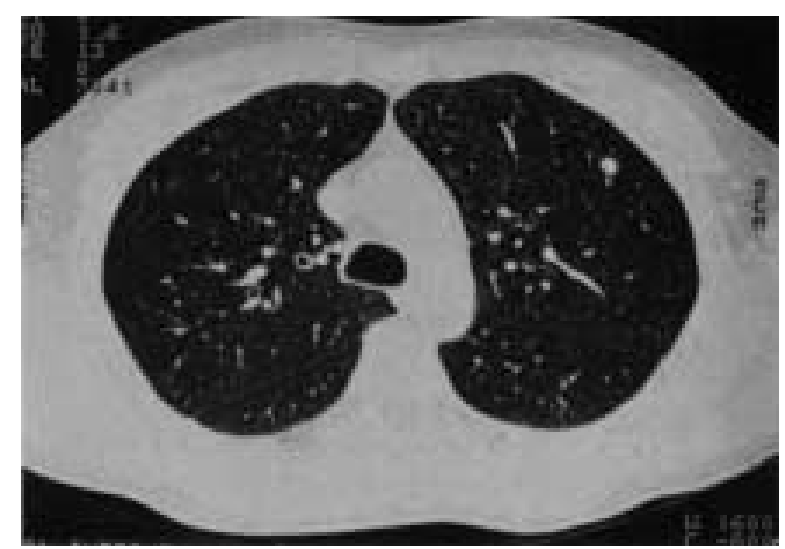

Fig. 1. - Computerized tomography showing an $8 \mathrm{~mm}$ spiculated tumour in the left upper lobe. pack-year history of cigarette smoking, and a chest Xray 3 yrs earlier (because of hyperventilation) had been normal. A chest radiograph showed an $8 \mathrm{~mm}$ nodule in the left upper lobe. This was confirmed by computed tomography (CT) (contiguous $10 \mathrm{~mm} \mathrm{CT}$ slices at inspiration through the whole lungs, and additionally $1 \mathrm{~mm}$ high resolution CT slices through the upper lobes (Somatom-plus, Siemens Erlangen, Germany)) to be noncalcified and with a spiculate outline (fig. 1). No other nodules or cysts, and no enlargement of mediastinal or hilar lymph nodes, were present. Pulmonary function tests revealed normal spirometry but a reduced transfer factor $(61 \%$ predicted).

Thoracotomy was performed for diagnostic wedge excision of the nodule. Frozen section revealed it to be a benign lesion, and the planned lobectomy was cancelled.

Histological examination showed a nodular interstitial infiltrate consisting mainly of clustered histiocytes with

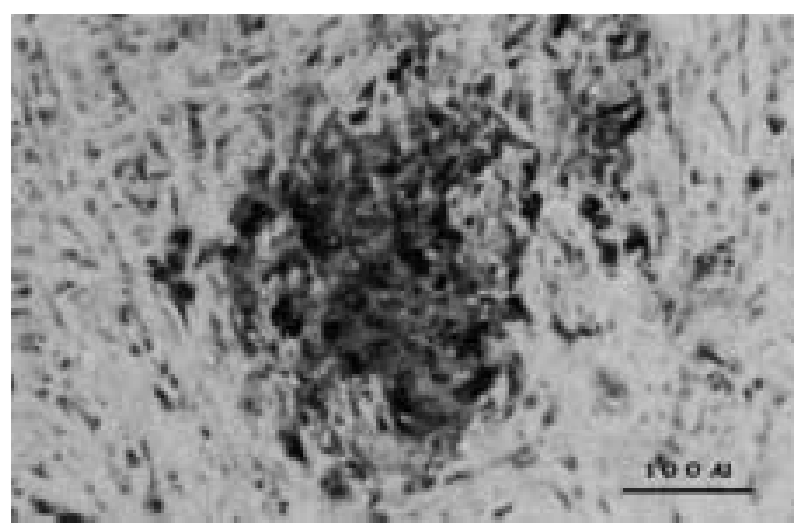

Fig. 2. - Small cluster of histiocytoid cells staining positively (dark) for $\mathrm{S}-100$. Bar=100 $\mu \mathrm{m}$. 
an admixture of eosinophilic granulocytes. Centrally the lesion showed slight dissolution. The histiocytes had a bland, folded or indented nucleus with eosinophilic cytoplasm. Immunohistochemical staining for S-100 was positive, identifying these cells as Langerhans' cells (fig. 2). Ultrastructural investigation showed them to contain Birbeck granules (Langerhans' bodies).

The definitive pathological diagnosis was eosinophilic granuloma of the lung. Further examination (bone scan) was normal. The patient has been followed-up for 3 yrs. During this time, the lung function has not changed, and, in particular, the transfer factor remained stable (66\% of predicted). Also, on chest X-ray, no further nodules or diffuse manifestations of eosinophilic granuloma have occurred.

\section{Discussion}

The evaluation and management of the SPN has recently been reviewed [5]. Although some features may suggest malignancy (gender, age, smoking history, growth of the SPN, or absence of the SPN on an earlier X-ray), the decision to perform thoracotomy in the individual patient is often difficult. Surprisingly, in our patient, the SPN proved to be a solitary eosinophilic granuloma.

The roentgenographic pattern of eosinophilic granuloma in the lung is usually diffuse and bilaterally symmetric, with a tendency to a predominance in the upper lung zones. In the early stage, the disease generally has a multinodular pattern, with individual lesions ranging from 1-10 $\mathrm{mm}$ in diameter [6]; a SPN has been described only once [4] but to our knowledge never as a sign of early stage of eosinophilic granuloma. Some patients show progression to a reticulonodular pattern or to an end-stage characterized by a very coarse reticular pattern. Unusual radiographic features such as hilar or mediastinal node enlargement, or pleural effusion have been reported [6].

Microscopically, cellular infiltrates are predominantly located in the interstitium around small bronchioles. Characteristic of the eosinophilic granuloma are Langerhans' cells, containing large irregularly folded nuclei, often with prominent nucleoli, and abundant pale eosinophilic cytoplasm. Electron microscopy shows cytoplasmic Langerhans' bodies (synonymous with Birbeck granules), consisting of microtubuli of parallel membranous structures showing a terminal expansion resulting in a "tennis racket like" appearance. These cells also show a positive reaction for protein S-100, which in clustered histiocytes only occurs in eosinophilic granuloma.

Treatment of eosinophilic granuloma is largely symptomatic, although corticosteroids and even chemotherapy have been tried without proven success.

The prognosis of eosinophilic granuloma is poor in patients with honeycombing and in patients with disseminated disease. If the disease is confined to the bones or the lungs, the outcome is considerably better [7], with spontaneous improvement or stabilization of the disease in most patients.

\section{References}

1. Siegelmann SS, Stilik FP, Summer WR. Management of the patient with a localized pulmonary lesion. In: Siegelmann SS, Stilik FP, Summer WR, eds. Pulmonary System: Practical Approaches to Pulmonary Diagnosis. New York, Grune and Stratton, 1979; pp. 339-358.

2. Toomes H, Delphendahl A, Hanke HG, Vogt-Moykopf I. The coin lesion of the lung. A review of 955 resected coin lesions. Cancer 1983; 51: 534-537.

3. Cummings SR, Lillington GA, Richard RJ. Estimating the probability of malignancy in solitary pulmonary nodules. A Bayesian approach. Am Rev Respir Dis 1986; 134: 449-452.

4. Fichtenbaum CJ, Kleinmann GM, Haddad RG. Eosinophilic granuloma of the lung presenting as a solitary pulmonary nodule. Thorax 1990; 45: 905-906.

5. Viggiano RE, Swensen SJ, Rosenow EC. Evaluation and management of solitary and multiple pulmonary nodules. Clin Chest Med 1992; 13: 83-95.

6. Fraser RG, Paré JAP, Paré PD, Fraser RS, Genereux GP (ed). Diagnosis of disease of the chest. Philadelphia, W.B. Saunders Co., 1991; pp. 2682-2693.

7. Freidman PJ, Liebour AA, Sokoloff J. Eosinophilic granuloma of the lung: clinical aspects of primary pulmonary histiocytosis in the adult. Medicine 1981; 60: 385-396. 